\title{
鼠 Practice Parameter: Evaluation and treatment of depression, psychosis, and dementia in Parkinson disease (an evidence-based review)
}

\author{
Report of the Quality Standards Subcommittee of the \\ American Academy of Neurology
}

\author{
J.M. Miyasaki, MD; K. Shannon, MD; V. Voon, MD; B. Ravina, MD, MSCE; G. Kleiner-Fisman, MD;
} K. Anderson, MD; L.M. Shulman, MD; G. Gronseth, MD; and W.J. Weiner, MD

\begin{abstract}
Objective: To make evidence-based treatment recommendations for patients with Parkinson disease (PD) with dementia, depression, and psychosis based on these questions: 1) What tools are effective to screen for depression, psychosis, and dementia in PD? 2) What are effective treatments for depression and psychosis in PD? 3) What are effective treatments for PD dementia or dementia with Lewy bodies (DLB)? Methods: A nine-member multispecialty committee evaluated available evidence from a structured literature review using MEDLINE, and the Cochrane Database of Health and Psychosocial Instruments from 1966 to 2004. Additional articles were identified by panel members. Results: The Beck Depression Inventory-I, Hamilton Depression Rating Scale, and Montgomery Asberg Depression Rating Scale should be considered to screen for depression in PD (Level B). The Mini-Mental State Examination and the Cambridge Cognitive Examination should be considered to screen for dementia in PD (Level B). Amitriptyline may be considered to treat depression in PD without dementia (Level C). For psychosis in PD, clozapine should be considered (Level B), quetiapine may be considered (Level C), but olanzapine should not be considered (Level B). Donepezil or rivastigmine should be considered for dementia in PD (Level B) and rivastigmine should be considered for DLB (Level B). Conclusions: Screening tools are available for depression and dementia in patients with PD, but more specific validated tools are needed. There are no widely used, validated tools for psychosis screening in Parkinson disease (PD). Clozapine successfully treats psychosis in PD. Cholinesterase inhibitors are effective treatments for dementia in $\mathrm{PD}$, but improvement is modest and motor side effects may occur.
\end{abstract}

NEUROLOGY 2006;66:996-1002

Statement of purpose. The Quality Standards Subcommittee (QSS) develops scientifically sound, clinically relevant practice parameters to guide the

Additional material related to this article can be found on the Neurology Web site. Go to www.neurology.org and scroll down the Table of Contents for the April 11 issue to find the title link for this article. practice of neurology. This article discusses treatments for the management of patients with depression, psychosis, and dementia in Parkinson disease (PD). These recommendations address the needs of neurologists and other clinicians caring for people with PD, patients and caregivers, research funding agencies, and researchers in movement disorders.

This article was previously published in electronic format as an Expedited E-Pub at www.neurology.org.

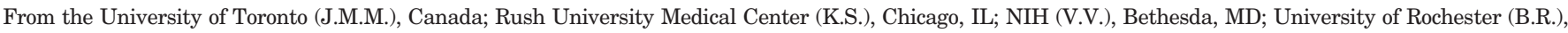
NY; University of Pennsylvania (G.K.-F.), Philadelphia; University of Maryland (K.A., L.M.S., W.J.W.), Baltimore; and University of Kansas (G.G.), Kansas City. Quality Standards Subcommittee Members are listed in appendix E-4 on the Neurology Web site at www.neurology.org.

Approved by QSS July 30, 2005; Practice Committee December 15, 2005; Board of Directors February $23,2006$.

Endorsed by the National Parkinson Foundation and the Parkinson's Disease Foundation.

Disclosures are provided after the text.

Received September 9, 2005. Accepted in final form February 16, 2006.

Address correspondence and reprint requests to the American Academy of Neurology, 1080 Montreal Avenue, St. Paul, MN 55116. 
This guideline provides answers to the following questions:

1. In patients with $\mathrm{PD}$, what are the most accurate tools to screen for depression, psychosis, and dementia?

2. In patients with $\mathrm{PD}$, what are the best treatments for depression and psychosis?

3 . What is the most effective treatment of dementia in PD or dementia with Lewy bodies (DLB)?

Background and justification. PD is the second most common neurodegenerative disease.$^{1}$ Characterized by the cardinal signs of bradykinesia, rigidity, tremor at rest, and abnormalities of balance, posture, and gait, the etiology of PD remains unknown in most patients. ${ }^{2}$ Nonmotor symptoms in PD, an increasingly recognized intrinsic feature of $\mathrm{PD}$, may affect three domains: autonomic, neuropsychiatric, and sensory, including pain. ${ }^{2}$ The prevalence of nonmotor symptoms is high. For instance, a survey of 99 patients with $\mathrm{PD}$ using validated questionnaires for nonmotor symptoms including anxiety, depression, sensory disturbance, fatigue, or sleep problems revealed that $88 \%$ of patients had at least one nonmotor symptom and $11 \%$ had five nonmotor symptoms. ${ }^{3}$ With improved treatment of motor symptoms, it is also now evident that the nonmotor features of PD such as dementia, depression, and psychosis may result in significant disability. ${ }^{2,4}$ Yet, despite the high prevalence and associated disability of nonmotor symptoms in $\mathrm{PD}$, physician recognition of these important clinical features is low. 5 Furthermore, many PD symptoms overlap with features of depression and dementia including symptoms of withdrawal, lack of motivation, flattened affect, decreased physical activity, or bradyphrenia, thus confounding the identification of these behavioral and cognitive disorders. It should be noted that validated criteria for depression, psychosis, and dementia in PD do not exist. Hence, the identification of clinically relevant screening and diagnostic tools for depression, psychosis, and cognitive decline validated specifically in the PD population is necessary.

In this parameter, the focus in the section on validation studies will be on the diagnostic accuracy of specific measures for behavioral disorders and dementia in PD. The mechanisms underlying nonmotor symptoms are poorly understood, and may be related to abnormalities of dopaminergic, serotonergic, adrenergic, cholinergic, and other peptidergic pathways. ${ }^{6-9}$ This complex pathophysiology reflects the resistance of nonmotor symptoms to dopamine replacement strategies. Therefore, specific treatments for autonomic, behavioral, and cognitive complications need to be employed.

Converging evidence suggests that the behavioral symptoms in PD may be pathophysiologically different from the behavioral symptoms observed in the general population. For instance, several lines of evidence suggest that PD depression may be related to the underlying pathology of PD itself rather than general psychiatric vulnerabilities and psychosocial associations. This suggests that reliance on the psychiatric treatment literature in the general population may not be sufficient and that specific treatment studies are required in PD.

The etiology of dementia in PD is unclear. Whether PD dementia represents a discrete categorical entity from DLB or exists on a spectrum is not known. For the purposes of this parameter, we will consider the treatments of both entities, presuming a similar underlying pathophysiology. ${ }^{10}$

Throughout this parameter, the term depression will be used to refer to major depression unless otherwise specified; there are no validation or treatment studies investigating forms of depression such as dysthymia or minor depression.

This parameter reviews the available evidence assessing diagnostic screening tools and the most effective treatments for dementia, depression, and psychosis in PD.

Description of the analytical process. The QSS of the American Academy of Neurology (AAN) identified a panel of six experienced movement disorder specialists, two psychiatrists, and a general neurologist with methodologic expertise. For the literature review, the following databases were searched: MEDLINE, EMBASE, CINAHL, the Cochrane Database of Systematic Reviews and Health and Psychosocial Instruments from 1966 to 2004. This was followed by a secondary search using the bibliography of retrieved articles and knowledge of the expert panel. Two authors reviewed each abstract for topic relevance. Two authors reviewed each full article to rate the level of evidence (Class I-IV) (appendices E-1 and E-2 on the Neurology Web site at www. neurology.org). If there was disagreement, the entire panel reviewed the article and the level of evidence was decided by consensus. The panel reviewed all articles cited in the evidence below. If a panelist was an author of one of the articles, at least two other panelists reviewed that article. Conflicts of interest were disclosed according to AAN guidelines. The AAN provided support and the Michael J. Fox Foundation funded the writing meetings. Panelists were not compensated.

Description of literature review. Search terms. Psychosis scale OR depression scale OR psychosis diagnosis OR depression diagnosis OR psychosis treatment OR depression treatment OR cognitive treatment OR dementia diagnosis OR psychoses OR hallucinations OR psychotic OR delusion OR depression OR depressive disorder OR adjustment disorder OR experimental drug therapy OR dementia treatment AND Parkinson disease OR diffuse Lewy body disease OR dementia with Lewy bodies.

Inclusion and exclusion criteria. For depression scales and treatment, Diagnostic and Statistical Manual (DSM) criteria for depression were the gold 
standard. DSM-IV criteria for major depression were used unless otherwise stated in the study reviewed. Various criteria for the diagnosis of PD were allowed. Class IV studies were not considered if Class III studies were available. Similarly, Class III studies were not considered if Class II studies were available. All Class I and II studies were included.

Depression screening tools. The search identified 37 articles. Thirty-four were rejected; 31 did not examine diagnostic accuracy and in 3 the patients did not have PD. Three articles were accepted (Class I, Class II).

Depression treatment (pharmacologic). The search identified 31 articles. Twenty were excluded because the populations studied were not PD patients with depression. Two were excluded because they were not randomized controlled trials. Nine articles were reviewed. An additional 27 articles were identified, 19 of which had been identified through the Cochrane bibliography. Of the 36 articles reviewed, 30 were rejected as they were Class IV articles. Six articles were accepted that were Class I, II, or III.

Depression treatment (nonpharmacologic). The search identified six studies: one Class II and five Class IV because of a high risk of bias. Class IV studies were not considered. One Class II study was accepted.

Psychosis screening tools. The search identified 31 articles. Eighteen did not examine diagnostic accuracy. Twelve articles did not include patients with PD. One Class IV article was accepted.

Psychosis treatment. The search identified 63 articles. Twenty-five were rejected because the patients did not have PD. Fifteen were rejected because the articles did not address psychosis treatment. Twenty-three articles received a full review. Eleven were rejected because they were Class III or IV. Three did not include patients with PD. Three were excluded because they were review articles, and one was excluded because it was an epidemiologic study. Four Class I and II articles were accepted.

Cognitive screening tools in PD. Twenty-four studies were identified. Ten were rejected because they did not examine diagnostic accuracy. One did not include patients with dementia. Thirteen articles received a full review; five did not examine diagnostic accuracy, five were Class IV, and one did not include PD patients with dementia. Two articles were accepted (Class I, III).

Cognitive treatment in PD or dementia with Lewy bodies. The search identified 331 articles. A total of 146 were excluded because they did not include patients with PD. A total of 115 were not randomized controlled trials. Forty-eight did not examine treatment for dementia. Twenty-two articles received a full review. An additional article was identified by the panelists and reviewed. Ten were Class III or IV. Five were excluded because they were review articles, and two articles were excluded because they included PD patients without dementia or criteria for dementia were not adequately defined. Three did not include cognitive treatment in PD. Three Class II articles were accepted.

Analysis of evidence. Question 1a: In patients with PD, which are the most accurate tools to screen for depression? Evidence. One Class I and two Class II articles compared the accuracy of depression screening tools to an independent reference standard based upon DSM criteria. ${ }^{11-13}$ These studies reported results of the Beck Depression Inventory (BDI), ${ }^{11}$ which is a self completion questionnaire (21 items, range 0-63), the Hamilton Depression Rating Scale (HDRS-17) (17 items, range 0-52), ${ }^{12,13}$ and the Montgomery Asberg Depression Rating Scale (MADRS) (10 items, range $0-60) .{ }^{12}$ Both the HDRS-17 and MADRS require a trained administrator and take 15 to 25 minutes each to administer. No studies examining the diagnostic accuracy of the Geriatric Depression Scale, Hospital Anxiety and Depression Scale, or Zung Self-Rating Depression Scale were identified.

All three studies were of prospective, cohort design. One employed a double masked methodology (Class I). ${ }^{11}$ The other two studies were not double masked (Class II). ${ }^{12,13}$ The authors reported various cut points and corresponding sensitivities and specificities for each screening tool. For the purposes of this article, we chose the cutpoint providing the greatest diagnostic accuracy for major depression (best specificity and sensitivity). For the BDI-I, a score of greater than 13 indicated depression, with a sensitivity of $67 \%$ (95\% CI 39 to 86 ) and a specificity of $88 \%$ (95\% CI 75 to 95 ). For the HDRS-17 (pooled results from two studies), a score of greater than 13 indicated depression, with a sensitivity of $83 \%$ (95\% CI 67 to 92) and specificity of 95\% (95\% CI 89 to 98). For the MADRS, the cut point was greater than 14 for patients indicating depression, with a sensitivity of $88 \%$ (95\% CI 64 to 97) and specificity of $89 \%$ (95\% CI 77 to 95). Although these data suggest that the HDRS-17 and MADRS are superior to the BDI, the studies were underpowered to determine superiority. In addition, the BDI is more easily administered, requiring at most 10 minutes.

Conclusions. For patients with PD, the BDI (one Class I) and HDRS (two Class II) are probably useful to screen for depression associated with PD. Based on one Class II study, MADRS is possibly useful to screen for depression associated with PD. Based on the available evidence, we cannot recommend one screening test over another.

There is insufficient evidence to support or refute the usefulness of other rating scales for depression in PD (Level U) (appendix E-3).

Recommendation. The BDI- I and HDRS should be considered for depression screening in PD (Level B). MADRS may be considered for screening for depression associated with PD (Level C). 
Question 1b: In patients with $P D$, which are the most accurate tools to screen for psychosis? Evidence. There is no gold standard for the diagnosis of psychosis in PD. There was one Class IV study ${ }^{14}$ in which an expert-derived Parkinson Psychosis Rating Scale (PPRS) was administered to 29 patients with PD and psychosis, and compared with two scales that have been validated in the general population: the Brief Psychosis Rating Scale (BPRS) and the Nurses Observation Scale for Inpatient Evaluation (NOSIEPsychotic). The PPRS demonstrated good interrater reliability and internal consistency. There was good diagnostic accuracy between the PPRS and BPRS $(p<0.01)$ and the PPRS and the NOSIE-Psychotic $(p<0.01)$. PD patients without psychosis were not included in the study, and therefore, the specificity of this screening tool in PD cannot be determined.

Conclusion. Based on one Class IV study, there is insufficient evidence to support or refute PPRS as a screening tool for psychosis in PD (Level U).

Recommendation. No recommendation is made.

Question 1c: In patients with PD, which are the most accurate tools to screen for dementia? Evidence. There was one Class $\mathrm{I}^{15}$ and one Class III study. ${ }^{16}$ In the Class I study, the Cambridge Cognitive Examination (CAMCog) and Mini-Mental State Examination (MMSE) were administered to 126 patients older than 60 years treated for PD in the community and institutions. Forty-four percent of this population had dementia by DSM-IV criteria. Both the CAMCog and MMSE had similar sensitivities (95\% and 98\%). However, the CAMCog was more specific (94\%) than the MMSE (77\%). The CAMCog includes all items of the MMSE and covers additional domains (orientation, concentration, expression, memory, abstract thinking, drawing, understanding, and writing), requiring approximately 20 minutes to administer by a trained rater.

In addition to scales, procedures are proposed to screen for PD dementia. In a case control EEG study (Class III) of 10 patients with PD dementia and 10 patients with PD without dementia, no significant differences in the amplitude of delta and theta activities were observed between the groups. ${ }^{16}$

Conclusion. The MMSE and CAMCog are probably useful for screening patients with PD and DSMdefined dementia (one Class I). The MMSE is as sensitive as the CAMCog and quicker to administer, but less specific.

Based on one Class III study, there is insufficient evidence to support the use of EEG as a screening tool for dementia in PD (Level U).

Recommendation. The MMSE and the CAMCog should be considered as screening tools for dementia in patients with $\mathrm{PD}$ (Level B).

Question 2: In patients with PD, what is the best pharmacologic treatment for depression? Evidence. Six studies were identified: one Class I, ${ }^{17}$ two Class II, ${ }^{18,19}$ and three Class III. ${ }^{20-22}$ All were randomized controlled trials. Interventions included amitriptyline, nortriptyline, citalopram, fluoxetine, sertraline, pergolide, pramipexole, and nefazodone. Three of the studies used placebo comparators. ${ }^{17-19}$ One study compared nefazodone to fluoxetine, ${ }^{22}$ one amitriptyline to fluoxetine,$^{20}$ and one pramipexole to pergolide. ${ }^{21}$ In four studies, depression was defined by DSM criteria. One study ${ }^{21}$ employed ICD-10 criteria for depression. In another study, the author's ad hoc scale was used. ${ }^{18}$ In all but one study, the severity of the depression was mild to moderate; depression was severe in the study of amitriptyline. ${ }^{20}$ Outcome measures varied and included BDI, HAM-D, MADRS, Zung Self Rating Depression Scale, and a unique rating scale. ${ }^{20}$

Five of the six studies used masked outcome assessment. The nefazodone vs fluoxetine study utilized independent but not masked outcome assessment (Class III). ${ }^{22}$ Three studies lacked allocation concealment of treatment groups (the attempt to prevent selection bias by concealing the assignment sequence until allocation to avoid maneuvering a patient to a particular assignment, either intentionally or unintentionally), ${ }^{20-22}$ one had nonstandard inclusion criteria, ${ }^{19}$ and one had less than $80 \%$ completers without an intent to treat analysis. ${ }^{20}$ Despite randomization, there were confounding differences in the severity of depression between groups in the pramipexole vs pergolide study (Class III). ${ }^{21}$ Follow-up ranged from 6 weeks to 12 months. The single Class I study, citalopram vs placebo, had the shortest duration of follow-up and used the HAMD for assessment. ${ }^{17}$

No significant benefit of treatment was observed in the studies of citalopram and sertraline. ${ }^{17,19} \mathrm{How}$ ever, neither study was sufficiently powered to exclude a clinically important benefit. Fluoxetine and nefazodone revealed equal efficacy for depression, but this study lacked a placebo control, and consequently, we could not conclude whether either drug was effective. ${ }^{22}$

Patients treated with pramipexole improved significantly more than patients treated with pergolide on measures assessing depression. ${ }^{21}$ However, there were important confounding differences in the severity of depression at baseline, which compromised these results.

In the study comparing the treatment of severely depressed patients with amitriptyline or fluoxetine, patients randomized to amitriptyline significantly improved (change in HAM-D of 14), while those treated with fluoxetine did not. ${ }^{20}$ Dropout rates were greater in the amitriptyline group due to adverse events.

In the nortriptyline study, the authors report a significant improvement in depression compared to placebo. ${ }^{18}$ However, it is impossible from the publication to determine if this difference was significant.

Conclusions. Based on one Class II study, amitriptyline is possibly effective in treating depression associated with PD. There is insufficient evidence to support or refute the efficacy of other specific antidepressants in the treatment of PD depression. Anti- 
cholinergic side effects, especially problematic with tricyclics, are an important consideration in the PD population due to concerns regarding potential worsening of cognition, as is the concern about orthostatic hypotension increasing the risk of falls.

Although the age at onset of PD is generally in adulthood, it should be noted that the Food and Drug Administration issued a drug labeling change in 2004 for a black box warning of the increased risk of suicidal ideation and suicide in adolescents and children with all antidepressants.

Recommendations. Amitriptyline may be considered in the treatment of depression associated with PD (Level C). Although the highest level of evidence is for amitriptyline, it is not necessarily the first choice for treatment of depression associated with PD. There is insufficient evidence to make recommendations regarding other treatments for depression in PD. Absence of literature demonstrating clear efficacy of non-tricyclic antidepressants is not the same as absence of efficacy.

Question 2b: In patients with PD and depression, what are the best nonpharmacologic treatments?

Evidence. No published trials of psychotherapy for depression associated with PD were available. The single Class II study randomized patients to transcranial magnetic stimulation (TMS) or fluoxetine. ${ }^{23}$ Outcomes were assessed in a blinded fashion using HAM-D. Completion rate was $100 \%$. A primary outcome measure was not specified. Both groups improved, but there was no difference in the magnitude of improvement in the treatment groups. The study was insufficiently powered to exclude a moderate difference in efficacy between the two therapies. Additionally, because of the absence of a placebo comparator, we cannot determine whether either intervention was effective. Due to these study design weaknesses, this study was downgraded to Class III evidence.

Only Class IV studies were available regarding ECT, which were not further evaluated.

Conclusion. There is insufficient evidence to support or refute the efficacy of TMS (single Class III) or ECT (Class IV) in the treatment of depression associated with PD (Level U).

Recommendation. No recommendations were made.

Question 2c. In patients with $P D$ and psychosis, what is the best treatment? Evidence. There were four randomized, double blind, controlled trials (one Class $\mathrm{I}^{24}$ and three Class $\left.\mathrm{II}^{25-27}\right)$. One study compared clozapine to quetiapine (Class II). ${ }^{25}$ Psychosis was defined using various criteria. Three studies were placebo controlled.

One Class I study demonstrated superiority of clozapine compared to placebo using the Clinical Global Impression Scale (CGI) $(p<0.001) .{ }^{24}$ This study also demonstrated improvement on the Brief Psychiatric Rating Scale (BPRS) $(p=0.002)$ and the Scale for the Assessment of Positive Symptoms (SAPS) $(p=$
0.01). Parkinsonism did not worsen and tremor improved. One patient discontinued due to leukopenia.

Two Class II studies compared olanzapine to placebo. ${ }^{26,27}$ In both studies, psychosis failed to improve and motor symptoms worsened.

One 12-week Class II study was randomized, open label, and used a blinded rater. Eleven patients received quetiapine and 12 received clozapine. ${ }^{25}$ Endpoints were change in BPRS, CGI, Unified Parkinson's Disease Rating Scale (UPDRS) motor subscore, and the Abnormal Involuntary Movement Scale (AIMS). BPRS improved by 9.1 ( $p<0.001)$ for quetiapine and 10.7 for clozapine $(p<0.001)$. For CGI, quetiapine improved by $1.5(p<0.001)$ and clozapine by $1.9(p<0.001)$. UPDRS motor worsened by $1.6(p=\mathrm{NS})$ for quetiapine and improved by 2.1 for clozapine $(p<0.005)$. AIMS improved by 1.6 for quetiapine $(p<0.05)$ and 1.8 for clozapine $(p<$ 0.05).

Conclusions. For patients with $\mathrm{PD}$ and psychosis, one Class I study and one Class II study demonstrated that clozapine is probably an effective treatment. Clozapine improved psychosis and resulted in improved motor function in some cases.

One Class II study demonstrated that quetiapine possibly improves psychosis in PD.

Two Class II studies demonstrated that olanzapine probably does not improve psychosis and worsens motor function.

There is a concern that all atypical neuroleptics have a small increased risk of mortality particularly in elderly patients with dementia who are treated for behavioral disorders. The mechanism for increased mortality is not clear. This must be balanced by the high morbidity and mortality associated with psychosis. ${ }^{2}$

Recommendations. For patients with PD and psychosis, clozapine should be considered (Level B). Clozapine use is associated with agranulocytosis that may be fatal. The absolute neutrophil count must be monitored. Monitoring requirements may vary according to country.

For patients with $\mathrm{PD}$ and psychosis, quetiapine may be considered (Level C).

For patients with PD and psychosis, olanzapine should not be routinely considered (Level B).

Question 3: what is the most effective treatment for dementia in $P D$ or DLB? Evidence. One Class I study was identified. ${ }^{28}$ The Class I study was a randomized, double-masked, placebo-controlled, crossover study in 22 subjects with PD and dementia. Each treatment period was 10 weeks separated by a 6 -week washout period. Donepezil was administered at 5 to $10 \mathrm{mg} /$ day. The primary outcome measure was the AD Assessment Scale-Cognitive Subscale (ADAScog). Donepezil was not significantly better than placebo based on ADAScog. Secondary endpoints (MMSE and CGI) were significantly better with donepezil. UPDRS scores did not deteriorate with donepezil.

Four Class II studies were identified. ${ }^{29-32}$ All stud- 
ies were randomized, controlled trials with blinded outcome assessments lasting 10 to 24 weeks. Three studies examined cholinesterase inhibitors (donepezil $^{30}$ rivastigmine ${ }^{29,31}$ ). One study examined piracetam, a compound of unknown mechanism of action. ${ }^{32}$ These studies employed DSM criteria for dementia. Primary outcome measures were change in the MMSE, ADAScog, AD Cooperative StudyClinicians Global Impression of Change (ADCSCGIC), the Clinicians Interview Based Impression of Change Plus Caregiver Input (CIBIC+), and a computerized cognitive assessment system speed score. Only one study focused on patients with DLB. ${ }^{31}$

When compared with placebo, piracetam did not show a significant benefit on any measure..$^{32}$ However, the study was insufficiently powered to exclude a moderate benefit of piracetam.

When rivastigmine $(\mathrm{n}=362)$ was compared with placebo $(\mathrm{n}=179)$, the ADAScog score improved 2.1 \pm 8.2 in the treatment group, but decreased by $0.7 \pm$ 7.5 in the placebo group $(p<0.001) .{ }^{29}$ The number needed to treat for any improvement as defined by the ADCS-CGIC was nine. The number needed to treat to obtain clinically meaningful (moderate or marked) improvement on the ADCS-CGIC was 19. Tremor increased in $10.2 \%$ vs $3.9 \%(p=0.01)$ in the treatment group. Sixty-two (17.1\%) patients on rivastigmine dropped out due to adverse events such as nausea, vomiting, and tremor. For every eight patients receiving rivastigmine, one patient dropped out due to adverse events. The number needed to harm was eight. This means that eight patients must experience worsening of parkinsonism as assessed by the UPDRS for each patient experiencing clinically meaningful improvement, as measured by the ADCS-CGIC.

Rivastigmine was evaluated in a randomized, double blind, placebo controlled trial of 120 patients with $\mathrm{DLB},{ }^{31}$ as defined by the DLB consensus guidelines. ${ }^{33}$ The intention to treat analysis of the primary outcome (computerized cognitive assessment system speed score) at week 20 revealed a benefit in the treatment group ( $p=0.048)$. At week 20 , there was no significant improvement in the MMSE or the Clinical Global Change-Plus. In the donepezil crossover design study $(\mathrm{n}=14)$, the MMSE improved by 2.1 (SD 2.7) compared to only 0.3 (SD 3.2) for placebo $(p=0.013) .{ }^{28}$ No change occurred in the UPDRS motor subscale scores. Two patients dropped out due to adverse events. On the CIBIC+, the number needed to treat to obtain any improvement was four. Number needed to harm was seven.

Conclusion. For patients with PD dementia or $\mathrm{DLB}$, rivastigmine is probably effective in improving cognitive function. However, the magnitude of the benefit is modest and tremor may be exacerbated (two Class II studies).

For patients with PD dementia, donepezil is probably effective in improving cognitive function. However, the magnitude of the benefits is modest (one Class I and one Class II study).
There is insufficient evidence to support or refute the efficacy of piracetam (Level U).Recommendations. Donepezil should be considered for the treat$\overline{\text { ment }}$ of dementia in PD (Level B).

Rivastigmine should be considered for the treatment of dementia in PD or DLB (Level B).

Recommendations for future research. Despite advances in treatment that improve motor symptoms for many patients, PD remains a progressive disease with complex, long-term, nonmotor symptoms that are often unrecognized. In order to identify the impact of depression, psychosis, and dementia, validated diagnostic questionnaires and rating scales are needed.

Depression rating scales. Current studies using the Beck Depression Inventory, Hamilton Scale for Depression, and the Montgomery Asberg Depression Rating Scale are underpowered to establish their diagnostic accuracy in this patient population. Other scales, such as the Geriatric Depression Scale and Zung Self-Rating Depression Scale, are not formally evaluated in PD. Future research is required to determine the best (sensitive, specific, but also practical for clinicians to rapidly administer) depression screening tool for patients with PD. DSM-IV criteria have not been validated for depression in PD.

Psychosis screening tools. Psychosis in PD is characterized by visual hallucinations and delusions (often paranoid). ${ }^{34}$ Screening tools for psychosis should be sensitive to hallucinations as well as other psychosis features such as delusions. Only one study evaluated the PPRS, ${ }^{15}$ which may be appropriate for patients with PD. ${ }^{15}$ However, in order to determine its specificity, the PPRS needs to be evaluated in nonpsychotic and psychotic PD patients. DSM-IV criteria for psychosis have not been validated in PD.

Cognition screening tools. Screening tools must be easy and quick to administer. Cognitive decline in $\mathrm{PD}$ is characterized by impaired executive function, visuospatial abnormalities, impaired memory, and language deficits. ${ }^{35}$ An appropriate scale that reliably incorporates executive function (e.g., frontal assessment battery and other practical tests of executive function) should be incorporated into a screening test for PD dementia. When evaluating new screening tools, the DSM-IV criteria for dementia may not be the most appropriate gold standard for patients with PD. DSM-IV criteria for dementia have not been validated in PD. In PD patients, it may be difficult to assess impairments in domains other than memory.

Depression treatment. There is a need for randomized, double-blinded, placebo-controlled studies of adequate size and duration of follow-up to assess antidepressants, psychotherapies, and other somatic therapies such as ECT and TMS.

Psychosis treatment. Due to rare but possible agranulocytosis and concerns about increased mortality associated with clozapine, other treatments should be identified for patients with PD and psycho- 
sis. Class I studies are required to evaluate the efficacy of quetiapine. Evidence for efficacy of novel antipsychotics without dopaminergic blocking effects is needed for effective treatment of psychosis in PD.

Dementia treatment. The cognitive benefits of donepezil and rivastigmine were small in PD dementia or DLB, and tremor increased with rivastigmine. Therefore, future research should include more Class I studies to assess the role of cholinesterase inhibitors and other medications in the treatment of dementia associated with PD. Additional treatments need to be developed that alleviate cognitive symptoms without worsening parkinsonism.

Disclaimer. This statement is provided as an educational service of the American Academy of Neurology. It is based on an assessment of current scientific and clinical information. It is not intended to include all possible proper methods of care for a particular neurologic problem or all legitimate criteria for choosing to use a specific procedure. Neither is it intended to exclude any reasonable alternative methodologies. The AAN recognizes that specific patient care decisions are the prerogative of the patient and the physician caring for the patient, based on all of the circumstances involved.

Disclosure. Dr. Miyasaki received research funds from Boehringer Ingelheim, Teva, and Janssen Ortho and consulting fees from Boehringer Ingelheim. Dr. Shannon received research funds from Teva. Dr. Shulman received research grants or unrestricted educational grants from Pfizer, Novartis, and Teva. Dr. Weiner received research grants from Teva, Boehringer Ingelheim, consultancy fees from Teva, and is a member of Boehringer Ingelheim's speakers bureau. Drs. Anderson, Ravina, and Gronseth have nothing to disclose.

\section{Acknowledgment}

The authors thank Nancy King and Wendy Edlund for administrative support and Andrew Wilner, MD, for help with manuscript preparation.

\section{References}

1. Van Den Eeden SK, Tanner CM, Bernstein AL, et al. Incidence of Parkinson's disease: variation by age, gender, and race/ethnicity. Am J Epidemiol 2003;157:1015-1022.

2. Factor S, Weiner W. Parkinson's disease. Diagnosis and clinical management. New York: Demos, 2002

3. Shulman LM, Taback RL, Bean J, Weiner WJ. Comorbidity of the nonmotor symptoms of Parkinson's disease. Mov Disord 2001;16:507510 .

4. Weintraub D, Moberg PJ, Duda JE, Katz IR, Stern MB. Effect of psychiatric and other nonmotor symptoms on disability in Parkinson's disease. J Am Geriatr Soc 2004;52:784-788.

5. Shulman LM, Taback RL, Rabinstein AA, Weiner WJ. Non-recognition of depression and other non-motor symptoms in Parkinson's disease. Parkinsonism Relat Disord 2002;8:193-197.

6. Agid Y, Taquet H, Cesselin F, Epelbaum J, Javoy-Agid F. Neuropeptides and Parkinson's disease. Prog Brain Res 1986;66:107-116.

7. Dubois B, Hauw JJ, Ruberg M, Serdaru M, Javoy-Agid F, Agid Y. [Dementia and Parkinson's disease: biochemical and anatomo-clinical correlation.] Rev Neurol (Paris) 1985;141:184-193.

8. Pillon B, Dubois B, Cusimano G, Bonnet AM, Lhermitte F, Agid Y. Does cognitive impairment in Parkinson's disease result from nondopaminergic lesions? J Neurol Neurosurg Psychiatry 1989;52:201-206.
9. Wakabayashi K, Takahashi H. Neuropathology of autonomic nervous system in Parkinson's disease. Eur Neurol 1997;38:2-7.

10. Noe E, Marder K, Bell KL, Jacobs DM, Manly JJ, Stern Y. Comparison of dementia with Lewy bodies to Alzheimer's disease and Parkinson's disease with dementia. Mov Disord 2004;19:60-67.

11. Leentjens AF, Verhey FR, Luijckx GJ, Troost J. The validity of the Beck Depression Inventory as a screening and diagnostic instrument for depression in patients with Parkinson's disease. Mov Disord 2000;15: 1221-1224.

12. Leentjens AF, Verhey FR, Lousberg R, Spitsbergen H, Wilmink FW. The validity of the Hamilton and Montgomery-Asberg depression rating scales as screening and diagnostic tools for depression in Parkinson's disease. Int J Geriatric Psychiatry 2000;15:644-649.

13. Naarding P, Leentjens AF, van Kooten F, Verhey FR. Disease-specific properties of the Rating Scale for Depression in patients with stroke, Alzheimer's dementia, and Parkinson's disease. J Neuropsychiatry Clin Neurosci 2002;14:329-334.

14. Friedberg G, Zoldan J, Weizman A, Melamed E. Parkinson Psychosis Rating Scale: a practical instrument for grading psychosis in Parkinson's disease. Clin Neuropharmacol 1998;21:280-284.

15. Hobson P, Meara J. The detection of dementia and cognitive impairment in a community population of elderly people with Parkinson's disease by use of the CAMCOG neuropsychological test. Age Ageing 1999;28:39-43.

16. Neufeld MY, Blumen S, Aitkin I, Parmet Y, Korczyn AD. EEG frequency analysis in demented and nondemented parkinsonian patients. Dementia 1994;5:23-28.

17. Wermuth L, Sorensen P, Timm S, et al. Depression in idiopathic Parkinson's disease treated with citalopram. Nord J Psychiatry 1998;52: 163-169.

18. Andersen J, Aabro E, Gulman N, Helmsted A, Pedersen H. Antidepressive treatment in Parkinson's disease. A controlled trial of the effect of nortriptyline in patients with Parkinson's disease treated with L-Dopa. Acta Neurol Scand 1980;62:210-219.

19. Leentjens AF, Vreeling FW, Luijckx GJ, Verhey FR. SSRIs in the treatment of depression in Parkinson's disease. Int J Geriatric Psychiatry 2003; 18:552-554

20. Serrano-Duenas M. Dosis bajas de amitriptilina frente a dosis bajas de fluoxetine en el tratamiento de la depression de enfermos con Parkinson. Rev Neurol 2002;35:1010-1014).

21. Rektorova I, Rektor I, Bares M, et al. Pramipexole and pergolide in the treatment of depression in Parkinson's disease: a national multicentre prospective randomized study. Eur J Neurol 2003;10:399-406.

22. Avilla A, Cardona X, Martin Baranera M, Maho P, Satre F, Bello J. Does nefazodone improve both depression and Parkinson disease? A pilot randomized trial. J Clin Psychopharmacol 2003;23:509-513.

23. Fregni F, Santos CM, Myczkowski ML, et al. Repetitive transcranial magnetic stimulation is as effective as fluoxetine in the treatment of depression in patients with Parkinson's disease. J Neurol Neurosurg Psychiatry 2004;75:1171-1174.

24. Parkinson Study Group. Low-dose clozapine for the treatment of druginduced psychosis in Parkinson's disease. N Engl J Med 1999;340:757763.

25. Morgante L, Epifanio A, Spina E, et al. Quetiapine versus clozapine: a preliminary report of comparative effects on dopaminergic psychosis in patients with Parkinson's disease. Neurol Sci 2002;23:S89-90.

26. Breier A, Sutton VK, Feldman PD, et al. Olanzapine in the treatment of dopamimetic-induced psychosis in patients with Parkinson's disease. Biol Psychiatry 2002;52:438-445.

27. Ondo W, Levy JK, Vuong KD, Hunter C, Jankovic J. Olanzapine treatment for dopaminergic-induced hallucinations. Mov Disord 2002;17: 1031-1035.

28. Ravina B, Putt M, Siderowf A, et al. Donepezil for dementia in Parkinson's disease: a randomized, double-blind, placebo controlled, crossover study. JNNP 2006 (in press).

29. Emre M, Aarsland D, Albanese A, et al. Rivastigmine for dementia associated with Parkinson's disease. N Engl J Med 2004;351:25092518.

30. Aarsland D, Laake K, Larsen JP, Janvin C. Donepezil for cognitive impairment in Parkinson's disease: a randomised controlled study. J Neurol Neurosurg Psychiatry 2002;72:708-712.

31. McKeith I, Del Ser T, Spano P, et al. Efficacy of rivastigmine in dementia with Lewy bodies: a randomised, double-blind, placebo-controlled international study. Lancet 2000;356:2031-2036.

32. Sano M, Stern Y, Marder K, Mayeux R. A controlled trial of piracetam in intellectually impaired patients with Parkinson's disease. Mov Disord 1990;5:230-234.

33. McKeith IG, Galasko D, Kosaka K, et al. Consensus guidelines for the clinical and pathologic diagnosis of dementia with Lewy bodies (DLB): report of the consortium on DLB international workshop. Neurology 1996; $47: 1113-1124$.

34. Weintraub D, Stern MB. Psychiatric complications in Parkinson disease. Am J Geriatr Psychiatry 2005;13:844-851.

35. Aarsland D, Andersen K, Larsen JP, Lolk A, Kragh-Sorensen P. Prevalence and characteristics of dementia in Parkinson disease: an 8-year prospective study. Arch Neurol 2003;60:387-392 


\section{Neurology}

Practice Parameter: Evaluation and treatment of depression, psychosis, and dementia in Parkinson disease (an evidence-based review): [RETIRED]: Report of the Quality Standards Subcommittee of the American Academy of Neurology J. M. Miyasaki, K. Shannon, V. Voon, et al. Neurology 2006;66;996-1002 Published Online before print April 2, 2006 DOI 10.1212/01.wnl.0000215428.46057.3d

\section{This information is current as of April 2, 2006}

\section{Updated Information \& Services}

Supplementary Material

\section{Citations}

Permissions \& Licensing

Reprints including high resolution figures, can be found at: http://n.neurology.org/content/66/7/996.full

Supplementary material can be found at: http://n.neurology.org/content/suppl/2006/04/06/66.7.996.DC1 http://n.neurology.org/content/supp1/2007/05/23/01.wnl.0000215428.4 6057.3d.DC2

http://n.neurology.org/content/suppl/2006/04/03/01.wnl.0000215428.4 6057.3d.DC1

This article has been cited by 17 HighWire-hosted articles: http://n.neurology.org/content/66/7/996.full\#\#otherarticles

Information about reproducing this article in parts (figures,tables) or in its entirety can be found online at:

http://www.neurology.org/about/about_the_journal\#permissions

Information about ordering reprints can be found online:

http://n.neurology.org/subscribers/advertise

Neurology ${ }^{\circledR}$ is the official journal of the American Academy of Neurology. Published continuously since 1951, it is now a weekly with 48 issues per year. Copyright . All rights reserved. Print ISSN: 0028-3878. Online ISSN: 1526-632X.

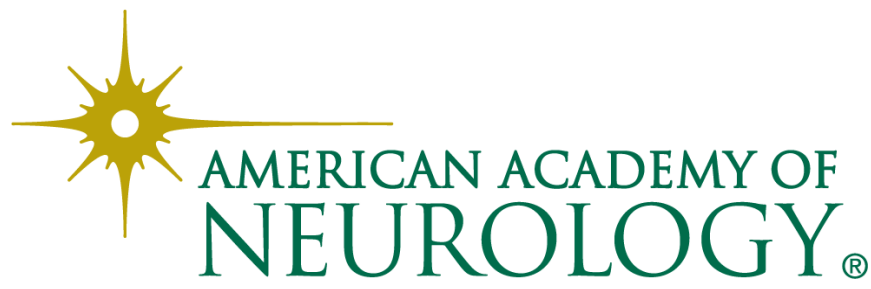

